\title{
Relationship between mechanical and magnetic properties in cold rolled low carbon steel
}

\author{
S. Takahashi, ${ }^{\text {a) }}$ S. Kobayashi, H. Kikuchi, and Y. Kamada \\ Nondestructive Evaluation and Science Research Center, Faculty of Engineering, Iwate University, Morioka \\ 020-8551, Japan
}

(Received 27 December 2005; accepted 13 October 2006; published online 12 December 2006)

\begin{abstract}
Structure-sensitive properties of minor hysteresis loops have been compared with Vickers hardness and ductile-brittle transition temperature (DBTT) obtained by Charpy impact test for cold rolled low carbon steel. Minor loops were measured with increasing magnetic field amplitude, step by step, and were analyzed in connection with the rolling reduction. We found that minor-loop coefficients deduced from relations between minor-loop parameters increase with increasing rolling reduction and have a simple relationship with both mechanical properties, Vickers hardness, and DBTT. We also found that these coefficients have a linear relation with coercive force obtained by the major loop. The present analysis method using minor loops is highly effective for nondestructive evaluation of ductile-brittle transition, in terms of the high sensitivity to lattice defects as well as low measurement field. (C) 2006 American Institute of Physics. [DOI: 10.1063/1.2401048]
\end{abstract}

\section{INTRODUCTION}

Nondestructive evaluation (NDE) of aging degradation of structural materials has been carried out for a long time. Various techniques using eddy current, ultrasonic wave, $\mathrm{x}$-ray, magnetic powder, etc., are now mature and widely used. Generally, these techniques are effective for detecting cracks with dimension greater than $0.1 \mathrm{~mm}$. Nevertheless, none of NDE techniques have been employed so far as a standard method to evaluate degradation before initiation of cracking.

Magnetic method is one of prominent candidates for NDE method to detect such degradation. It is well-known that the hysteresis loop is strongly affected by lattice defects such as dislocations, grain boundaries, impurity atoms, and precipitates. ${ }^{1-3}$ Both experimental and theoretical works revealed simple relationships between magnetic properties and dislocation density that both coercive force and inverse of initial susceptibility increase in proportion to the square root of dislocation density $\rho$. Such relations between magnetism and lattice defects in ferromagnetic materials were recently summarized by Kronmüller and Fähnle in their book. ${ }^{1}$

Minor hysteresis loops, which are obtained at magnetic fields less than saturation field, also contain much information about lattice defects, although minor-loop properties strongly depend on magnetic field amplitude $H_{a}$ and their physical meaning is vague. ${ }^{4}$ Recently, we analyzed a set of minor loops in $\mathrm{Fe}$ single crystals, ${ }^{5,6}$ polycrystals, ${ }^{7}$ and A533B steels, ${ }^{8}$ and found that there exist simple relations between minor-loop properties. We found that minor-loop coefficients deduced from these relations are independent of $H_{a}$ and are more sensitive to lattice defects than the traditional structure-sensitive properties of the major loop. This analysis shows that minor loops can be applicable to NDE of ferromagnetic steels.

Heavy dose of neutron radiation gives rise to the degra-

a)Electronic mail: seiki.t@iwate-u.ac.jp dation of pressure vessel steels before crack initiation. The neutron radiation produces high density of precipitates, point defects, and dislocation loops, and hence remarkably decreases the ductility of materials. ${ }^{9}$ At present, Charpy impact test is only the method for testing the degradation in reactor pressure vessels and is the most reliable test for ductilebrittle transition. The reactors are usually designed for 40 years of service, but they are now planned to extend their lifetime to 60 years. The integrity of pressure vessels is evaluated by Charpy impact test that is a destructive testing. The lack of Charpy impact test samples preinstalled in the nuclear reactor becomes an urgent issue.

Of our interest is a comparison of ductile-brittle transition temperature (DBTT) determined by Charpy impact test with the structure-sensitive magnetic properties. DBTT can be described by dislocation mobility when the hammer collides with the sample. In the brittle state, dislocations cannot be displaced owing to obstacles, while in the ductile state above DBTT, dislocations can overcome the obstacles with the help of thermal activation energy. DBTT depends on the obstacles against the dislocation displacement and is therefore sensitive to the degradation of steels. In the present study, dislocations were introduced as obstacles by cold rolling in low carbon steel and the relationship between DBTT and structure-sensitive properties of major and minor loops was investigated in detail. The results would be helpful for NDE of pressure vessels in the practical application, though obstacles against dislocations may be different from those produced by the irradiation damage.

\section{ANALYSIS METHOD OF MINOR HYSTERESIS LOOPS}

Preisach model has been widely used to analyze minor loops. However, building Preisach function needs some effort. In order to simplify the analysis, Tomás proposed an analysis method by using a differential susceptibility matrix calculated from minor-loop measurements. ${ }^{4}$ 


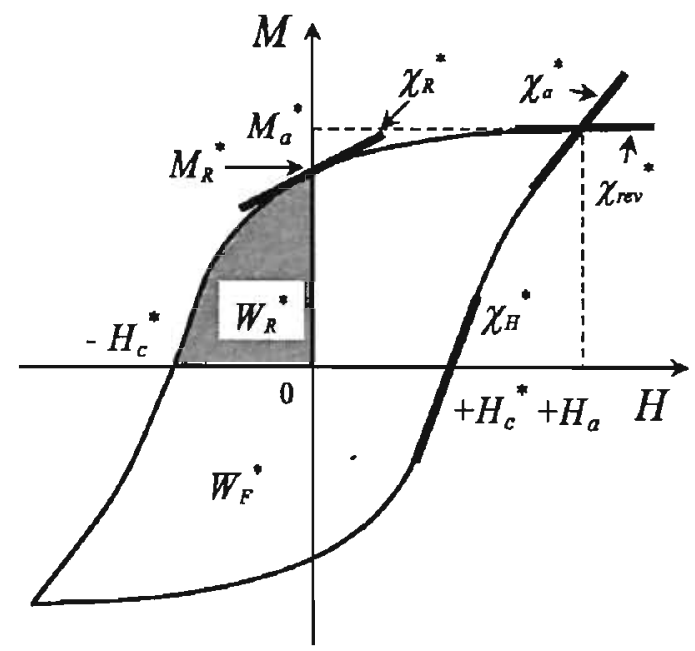

FIG. 1. Magnetic properties of a minor hysteresis loop.

Our analysis method of minor loops is based on our previous experimental results for $\mathrm{Fe}$ single crystals ${ }^{5,6}$ and polycrystals. ${ }^{7}$ As shown in Fig. 1, several minor-loop properties are introduced; minor-loop magnetization $M_{a *}^{*}$, minorloop coercive force $H_{c}{ }^{*}$, minor-loop remanence $M_{R}{ }^{*}$, minorloop hysteresis loss $W_{F}{ }^{*}$, minor-loop remanence work $W_{R}{ }^{*}$, and three minor-loop susceptibilities $\chi_{H}{ }^{*}, \chi_{R}{ }^{*}$, and $\chi_{a}{ }^{*}$ at $H$ $=H_{c}^{*}, 0$, and $H_{a}$, respectively.

The magnetization process before saturation can be divided conveniently into three stages. With increasing $H_{a}$ after demagnetizing the sample, $M_{a}{ }^{*}$ shows a gentle increase in the first stage and then steeply increases in the second stage, followed by a gradual increase of $M_{a}{ }^{*}$ toward the saturation in the third stage. The most part of magnetization proceeds in the second stage through the movement of Bloch wall. We found that there exist several rules between a pair of minorloop properties in the first or second stage as follows: ${ }^{7}$

$$
\begin{aligned}
& W_{F}^{*}=W_{F}^{0}\left(\frac{M_{a}^{*}}{M_{S}}\right)^{n_{F}}, \\
& W_{R}^{*}=W_{R}^{0}\left(\frac{M_{R}^{*}}{M_{R}}\right)^{n_{R}}, \\
& {H_{C}}^{*}=H_{C}^{0}\left(\frac{M_{R}^{*}}{M_{R}}\right)^{n_{C}}, \\
& \chi_{R}^{*}=\chi_{T}^{0}\left(\frac{M_{R}^{*}}{M_{R}}\right)^{n_{T}}, \\
& \chi_{a}^{*}=\chi_{S}^{0}\left(\frac{M_{a}^{*}}{M_{S}}\right)^{n_{S}},
\end{aligned}
$$

in the second stage, and

$$
R_{C}{ }^{*}=\frac{1}{\chi_{H}{ }^{*}}=R_{C}{ }^{0} \exp \left(-b \frac{H_{C}{ }^{*}}{H_{C}}\right),
$$

in the first stage. Here, $W_{F}{ }^{0}, W_{R}{ }^{0}, H_{c}{ }^{0}, \chi_{T}{ }^{0}, \chi_{s}{ }^{0}$, and $R_{c}{ }^{0}$ are minor-loop coefficients which are sensitive to lattice defects such as dislocations and grain boundaries, and are indepen-
TABLE I. Chemical compositions of S15C steel.

\begin{tabular}{ccccc}
\hline \hline S15C & $\mathrm{C}(\mathrm{wt} \%)$ & $\mathrm{Si}(\mathrm{wt} \%)$ & Mn (wt \%) & Fe (wt \%) \\
\hline 0.16 & 0.20 & 0.44 & Balance \\
\hline \hline
\end{tabular}

dent of $H_{a} . M_{S}$ and $M_{R}$ are the saturation magnetization and remanence of the major loop, respectively. The exponents $n_{F}$ and $n_{R}$ are nearly 1.5 being independent of materials, while $n_{c}, n_{T}$, and $n_{S}$ are a positive value less than 1.0 and slightly depend on the kinds of materials. Steinmetz studied the relation between $W_{F}^{*}$ and $M_{a}^{*}$ about a century ago and reported that $n_{F}$ is about 1.6 in the second stage. ${ }^{10}$ This relation is well-known as Steinmetz law.

The minor-loop coefficients have useful characters for NDE in terms of the high sensitivity to lattice defects as well as the low applied field required for measurements. Also, coercive force of the major loop is useful property related to dislocations. In this paper, we present the comparison between minor-loop coefficients and coercive force in connection with cold rolling reduction.

\section{EXPERIMENTAL PROCEDURE}

Magnetic properties both of major and minor loops were compared with mechanical properties, i.e., Vickers hardness and DBTT determined by Charpy impact test for cold rolled low carbon "S15C" steel. The chemical compositions of S15C steel are listed in Table I.

The material was annealed at $1123 \mathrm{~K}$ for $1 \mathrm{~h}$, followed by cold rolling with $0 \%-40 \%$ reduction at room temperature. Microstructures of dislocations before and after cold rolling were observed with transmission electron microscope (TEM). The samples were then cut for magnetic and mechanical measurements as shown in Fig. 2.

Charpy impact test was performed with a pendulum of $27.6 \mathrm{kG}$ and lift angle of $138.5^{\circ}$ at various temperatures ranging from 200 to $360 \mathrm{~K}$. The process of Charpy impact test was automatically executed by the robot, which guarantees the reproducibility of the data. Five Charpy samples were tested at each temperature, and both the largest and smallest values were eliminated when averaging the data. Vickers hardness was measured with load of $300 \mathrm{~g}$. Ten mea-
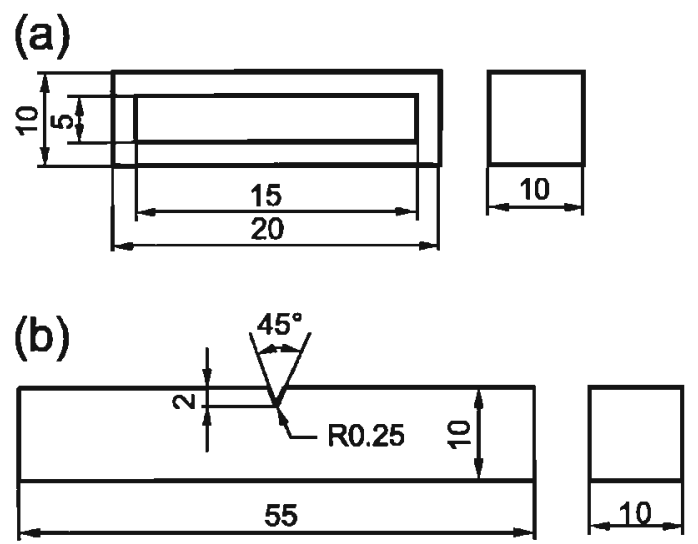

FIG. 2. Size and shape of samples for (a) magnetic measurements and (b) Charpy impact test. The dimensions are in millimeters. 


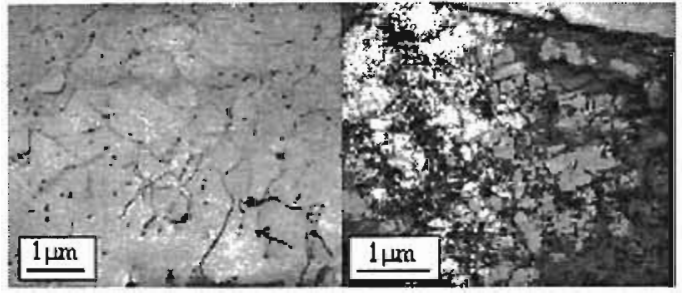

(a)

(b)

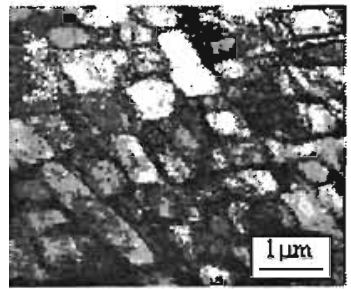

(c)

FIG. 3. TEM micrographs showing dislocations in S15C steel with (a) $0 \%$, (b) $5 \%$, and (c) $20 \%$ rolling reduction.

surements were carried out for each sample, and both the largest and smallest values were eliminated for averaged data.

Magnetic measurements were performed at room temperature for a picture-frame sample wound with 180 turn exciting and 20 turn detecting coils. After demagnetizing the sample, minor loops were measured, step by step, with increasing $H_{a}$, while the major loop was obtained with $H_{a}$ $=4 \mathrm{kA} / \mathrm{m}$.

\section{EXPERIMENTAL RESULTS}

As is seen in TEM micrographs in Fig. 3, $\rho$ increases with rolling reduction. Before the cold rolling, $\rho$ is low and dislocations are distributed homogenously as shown in Fig. 3(a). After $20 \%$ reduction, dislocation cells are formed and $\rho$ becomes to be more than $10^{10} \mathrm{~cm}^{-2}$ as shown in Fig. 3(c).

Figure 4 shows absorption energy as a function of temperature, obtained by Charpy impact test. For samples with no cold rolling, the absorption energy changes from $183 \mathrm{~J}$ at $T=333 \mathrm{~K}$ to $4.5 \mathrm{~J}$ at $T=201 \mathrm{~K}$, and DBTT is estimated to be $246 \mathrm{~K}$. On the other hand, for samples with $40 \%$ rolling reduction, the absorption energy at $333 \mathrm{~K}$ decreases by $40 \%$

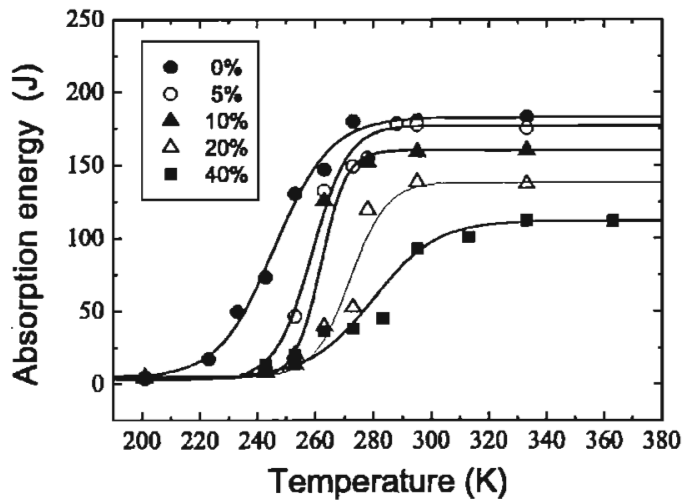

FIG. 4. Absorption energy obtained by Charpy impact test for S15C steel with and without rolling reduction.

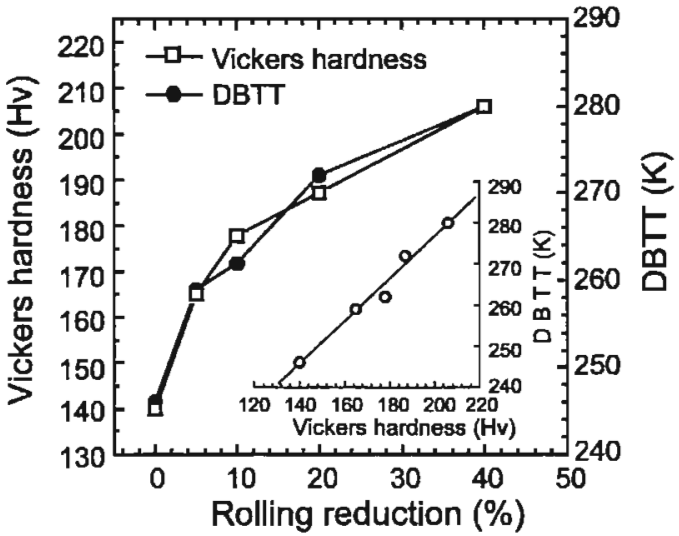

FIG. 5. Vickers hardness and ductile-brittle transition temperature (DBTT) as a function of rolling reduction for $\mathrm{S} 15 \mathrm{C}$ steel. The inset shows the relation between these mechanical properties.

and DBTT increases remarkably by $35 \mathrm{~K}$. The dependence of DBTT and Vickers hardness on rolling reduction is shown in Fig. 5. Both mechanical properties increase with rolling reduction and show almost the same behavior with each other.

Figures 6-9 show the relations between minor-loop properties, i.e., between $W_{F}{ }^{*}$ and $M_{a}{ }^{*}$, between $W_{R}{ }^{*}$ and $M_{R}{ }^{*}$, between $H_{c}{ }^{*}$ and $M_{R}{ }^{*}$, and between $R_{c}{ }^{*}\left(=1 / \chi_{H}{ }^{*}\right)$ and $H_{c}^{*}$, for various rolling reduction, respectively. As can be seen in the linear relation in the double logarithmic plot, the relations of Figs. $6-8$ are well represented by Eqs. (1)-(3), respectively, in the second stage, while the relation between $R_{c}^{*}$ and $H_{c}^{*}$ is expressed by Eq. (6) in the first stage. Similarly, the relations between $\chi_{R}{ }^{*}$ and $M_{R}{ }^{*}$ and between $\chi_{a}{ }^{*}$ and $M_{a}^{*}$ were well fitted to Eqs. (4) and (5), respectively, in the second stage. These least-squares fits yielded minor-loop constants of $n_{F}=1.59 \pm 0.01, n_{R}=1.53 \pm 0.04, n_{c}=0.44 \pm 0.04$, $n_{T}=0.22 \pm 0.01, n_{s}=0.66 \pm 0.07$, and $b=3.8 \pm 0.3$ as well as minor-loop coefficients as a function of rolling reduction as shown in Fig. 10. One can clearly see that all minor-loop coefficients $W_{F}^{0}, W_{R}^{0}, H_{c}^{0}, 1 / \chi_{T}^{0}, 1 / \chi_{s}{ }^{0}$, and $R_{c}{ }^{0}$ increase

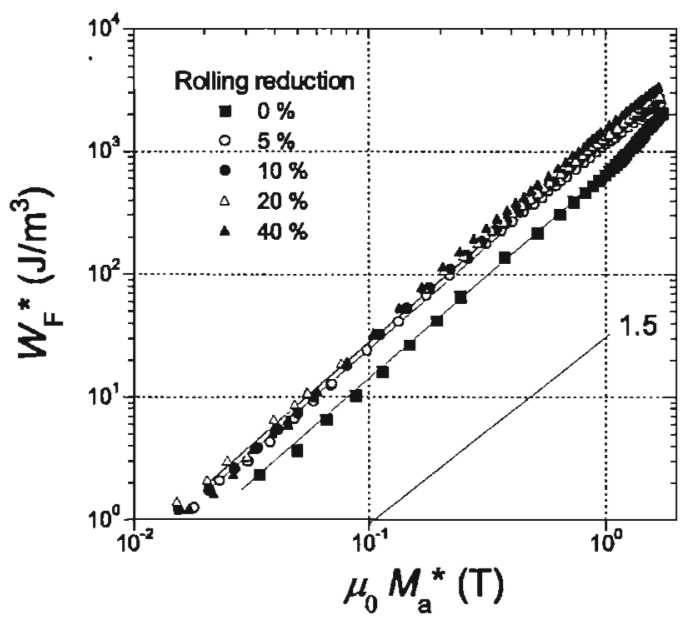

FIG. 6. Relation between $W_{F}^{*}$ and $M_{a}^{*}$ for S15C steel with and without rolling reduction. The solid lines through the data denote least-squares fits in the second stage. 


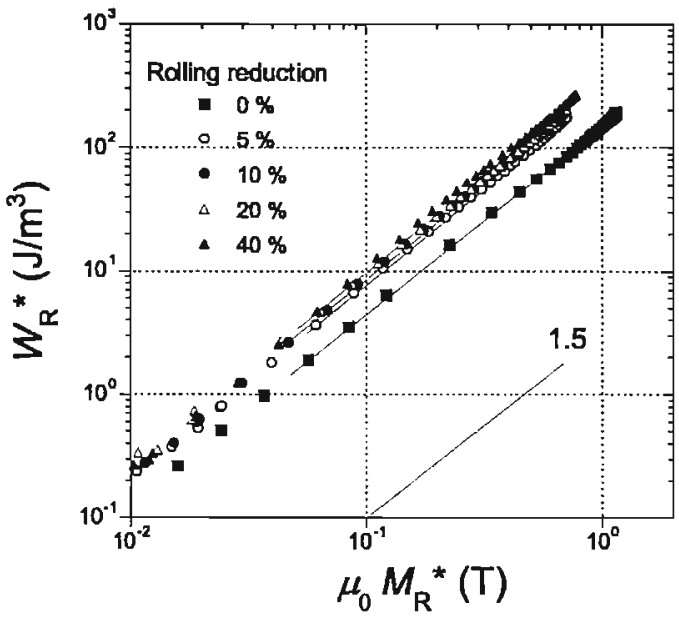

FIG. 7. Relation between $W_{R}^{*}$ and $M_{R}{ }^{*}$ for S15C steel with and without rolling reduction. The solid lines through the data denote least-squares fits in the second stage.

with increasing rolling reduction and show the similar dependence to coercive force of the major loop $H_{c}$.

\section{DISCUSSION}

Two mechanical properties, Vickers hardness and DBTT, obtained by macroscopic measurements, agree with each other as shown in Fig. 5, although Vickers hardness and DBTT reflect mechanical properties near the sample surface and inside the sample, respectively. Since the rolling reduction corresponds to the value of $\rho$, this agreement suggests that the value of $\rho$ on the surface has a linear relation with that inside the sample irrespective of the spatial distribution of dislocations.

DBTT gives us the direct information about ductility and brittleness from a macroscopic viewpoint. On the other hand, DBTT can be explained by the dislocation theory from a microscopic viewpoint. In Charpy impact test above DBTT, the greater part of the potential energy of a hammer is changed into kinetic energy of dislocations and the plastic deformation occurs through dislocation movement, while in

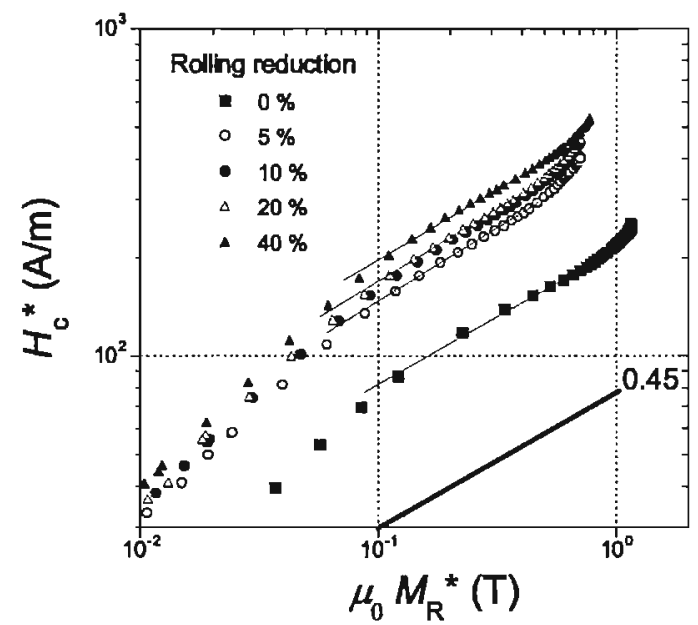

FIG. 8. Relation between $H_{c}{ }^{*}$ and $M_{R}{ }^{*}$ for S15C steel with and without rolling reduction. The solid lines through the data denote least-squares fits in the second stage.

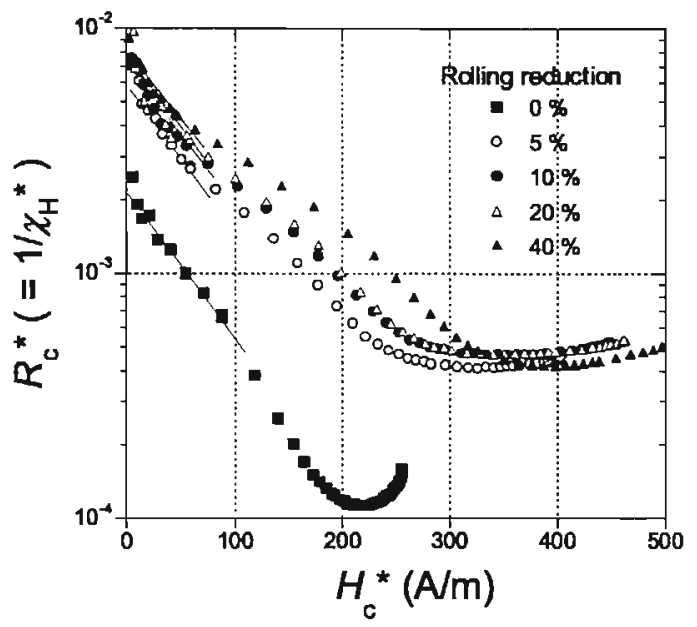

FIG. 9. Relation between $R_{c}{ }^{*}$ and $H_{c}{ }^{*}$ for S15C steel with and without rolling reduction. The solid lines through the data denote least-squares fits in the first stage.

the brittle state below DBTT, only small part of the potential energy is transferred into the kinetic energy. For cold rolled samples, DBTT depends on the number and distribution of dislocations and becomes higher with the increase of $\rho$.

Vickers hardness reflects the mean free path of dislocation movement. The depth of an indentation depends on dislocation mobility under constant local pressure. Since dislocations introduced by cold rolling play as obstacles and restrict the dislocation displacement, Vickers hardness increases with increasing $\rho$. This microstructural mechanism of dislocation movement, which is similar to that in Charpy impact test, yields a linear relationship between DBTT and Vickers hardness as shown in the inset in Fig. 5.

On the other hand, dislocations interact with magnetic domain walls and influence their movement. According to earlier theory for micromagnetism, arrangement of magnetization is determined so as to minimize magnetic Gibbs free energy consisting of exchange energy, magnetocrystalline anisotropy energy, magnetostatic energy, and magnetoelastic energy. ${ }^{1}$ In ferromagnetic material including dislocations, the Gibbs free energy is lowered when domain walls are located at dislocations and dislocations act as obstacles for the domain wall motion. This indicates that magnetic properties

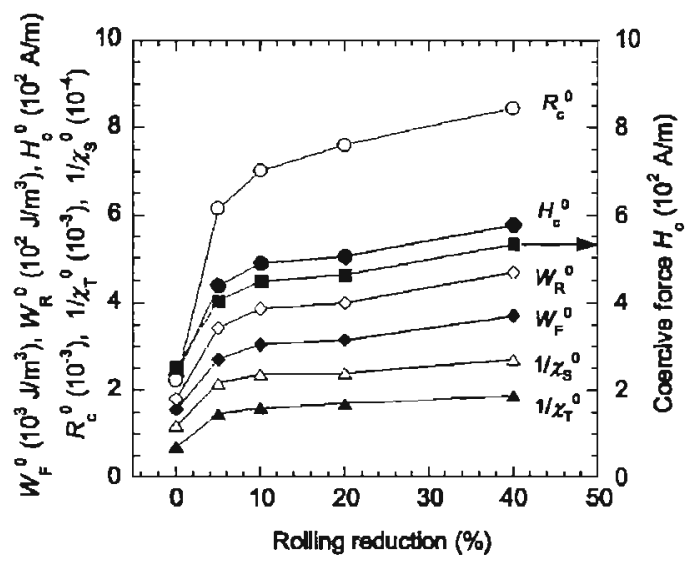

FIG. 10. Minor-loop coefficients and coercive force as a function of rolling reduction for $\mathrm{S} 15 \mathrm{C}$ steel. 

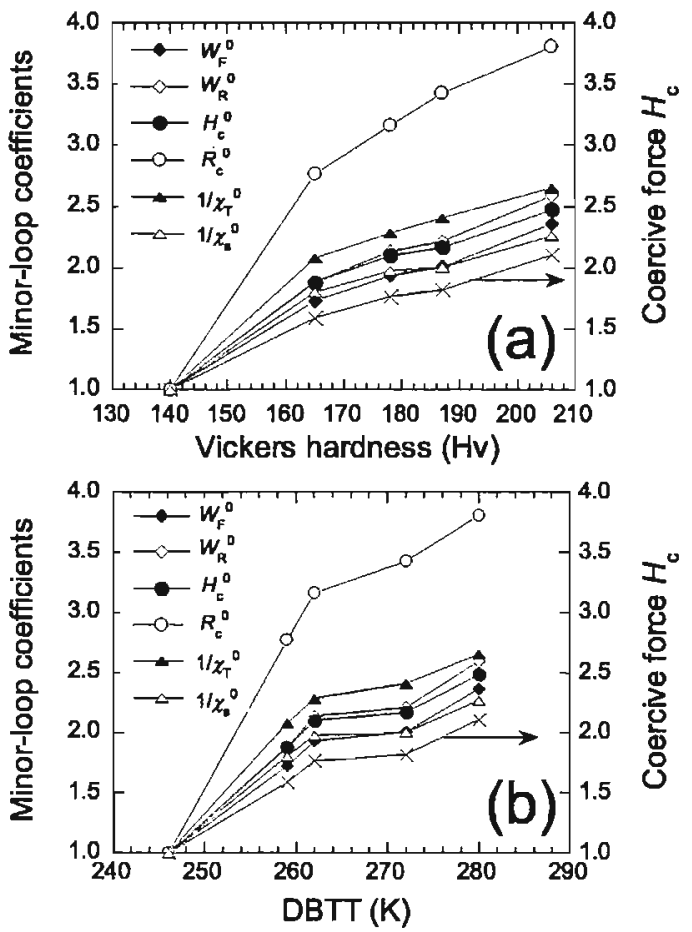

FIG. 11. Relations of magnetic properties with (a) Vickers hardness and (b) DBTT for S15C steel. The data are normalized to their initial value.

change with the increase of $\rho$ and both magnetic and mechanical properties should have an intimate connection with each other through dislocations. Actually, both minor-loop coefficients and $H_{c}$ show a simple relation both with Vickers hardness and DBTT as shown in Fig. 11. Note that because of the experimental difficulty of obtaining an accurate value of DBTT, the relation of Fig. 11(b) does not show a smooth change. Nevertheless, considering a linear relation between DBTT and Vickers hardness shown in the inset in Fig. 5, we conclude that DBTT is also simply related with minor-loop coefficients and $H_{c}$.

Structure-sensitive magnetic properties depend on the density and distribution of dislocations. It was revealed experimentally and theoretically that $H_{c}$ increases in proportion to the square root of $\rho{ }^{1}$ Since $H_{c}$ increases by two times due to $40 \%$ rolling reduction, the value of $\rho$ would increase by four times at most. However, the TEM observation shows that the increase of $\rho$ is much more than four times even for $20 \%$ rolling reduction as shown in Fig. 3. Such a large difference in the estimated value of $\rho$ would be attributed to the underestimate of $\rho$ for the initial state by the TEM observation, because the TEM observation is limited to very small local area while $H_{c}$ gives the information on $\rho$ averaged over whole sample. In fact, using the value of $H_{c}$ for the initial state, $\rho$ for the initial state was estimated to be $\sim 10^{10} \mathrm{~cm}^{-2}$, which is of the same order of magnitude as $\rho$ for $20 \%$ rolling reduction. Nevertheless, our TEM observation indicates that the cell structure grows by cold rolling and both the cell structure and $\rho$ contributes to the change in magnetic properties effectively.

Both structure-sensitive magnetic properties and DBTT are macroscopic properties. While the former is the property averaged over whole sample, the later is the local mechanical

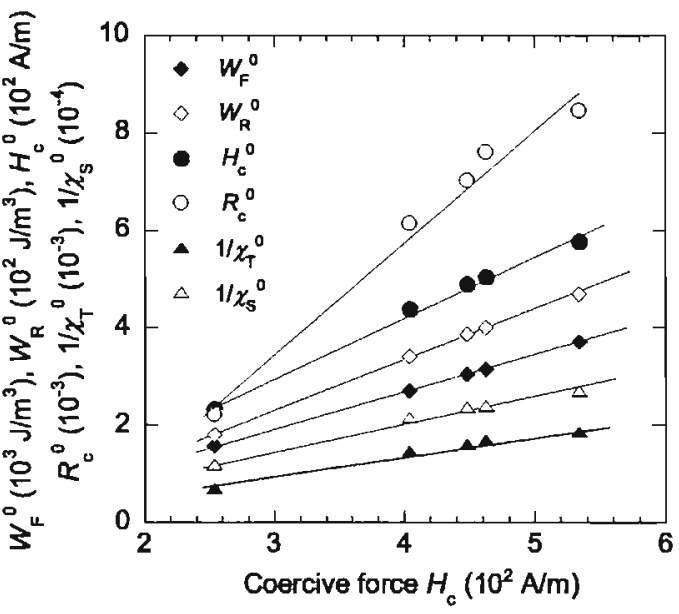

FIG. 12. Relation between minor-loop coefficients and coercive force for S15C steel.

property near the notch of the sample. If dislocations are distributed homogeneously inside the sample, these two properties have the same source in dislocations. The crucial difference between them is the fact that magnetic properties can be obtained by nondestructive measurements while Charpy impact test is destructive one. The simple relationship of two properties suggests that the practical application of magnetic methods to NDE can be expected for the pressure vessel of nuclear reactors. The neutron damage causes various kinds of lattice defects such as vacancies, interstitial atoms, voids, dislocation loops, cooper precipitates, etc., which disturb dislocation movement and make the material brittle. These lattice defects will interact with domain walls as in the case of dislocations. ${ }^{1-3}$ Quite recently, we performed in situ magnetic measurements under neutron radiation in Fe metal and pressure vessel steel and analyzed minor loops in relation to these radiation defects. " It was found that the present analysis method for dislocations using Eqs. (1)-(6) is also applicable for radiation defects and that minor-loop coefficients change during neutron irradiation both in Fe metal and A533B steels. These results confirmed the applicability of the magnetic method to NDE of pressure vessels and further studies for neutron-irradiated steels will appear elsewhere.

Finally, we discuss the relation between minor-loop coefficients and coercive force. As shown in Fig. 12, minorloop coefficients have a linear relation with $H_{c}$. This indicates that the minor-loop coefficients are connected simply with $H_{c}$ and with each other. From the viewpoint of the potential of domain wall displacement, $H_{c}$ gives us information on the highest crest of potential, while the minor-loop coefficients include mean values of the crest height, the trough depth, and the mobility of domain walls at the special point of potential; ${ }^{7} W_{F}^{0}, W_{R}^{0}$, and $H_{c}^{0}$ give information on the size and shape of potential, and $\chi_{T}{ }^{0}, \chi_{s}{ }^{0}$, and $R_{c}{ }^{0}$ related to the minor-loop susceptibility depend on the mobility of domain walls. The minor-loop coefficients increase by $2.4-3.8$ times due to $40 \%$ rolling reduction, whereas $H_{c}$ increases by 2.1 times. The difference in sensitivity indicates that dislocations exert a larger influence on the lower part of potential than the 
highest crest of potential and that minor loops are strongly influenced by the distribution of dislocations.

\section{CONCLUSION}

Ductile-brittle transition temperature and Vickers hardness have been compared with structure-sensitive magnetic properties of major and minor loops for cold rolled low carbon steel. Coercive force as well as minor-loop coefficients obtained from relations between minor-loop parameters were found to increase with rolling reduction and show a simple relationship with these mechanical properties. Moreover, the minor-loop coefficients have a linear relation with coercive force and have several useful characteristics in terms of high sensitivity to lattice defects, amount of information, and low magnetic field required for measurements. These results strongly indicate that the magnetic method seems to be the highly probable one for NDE of nuclear pressure vessels instead of Charpy impact test.

\section{ACKNOWLEDGMENT}

This research was supported by a Grant-in-Aid for Scientific Research (S), Grant No. 141020345, from the Ministry of Education, Culture, Sports, Science and Technology of Japan.

${ }^{1} \mathrm{H}$. Kronmüller and M. Fähnle, in Micromagnetism and the Microstructure of Ferromagnetic Solids (Cambridge University Press, Cambridge, 2003).

${ }^{2}$ L. J. Dijkstra and C. Wert, Phys. Rev. 79, 979 (1950).

${ }^{3}$ J. B. Goodenough, Phys. Rev. 95, 917 (1954).

${ }^{4}$ I. Tomás, J. Magn. Magn. Mater. 268, 178 (2004).

${ }^{5}$ S. Takahashi, L. Zhang, and T. Ueda, J. Phys.: Condens. Matter 15, 7997 (2003).

${ }^{6}$ S. Takahashi, T. Ueda, and L. Zhang, J. Phys. Soc. Jpn. 73, 239 (2004).

${ }^{7}$ S. Takahashi and L. Zhang, J. Phys. Soc. Jpn. 73, 1567 (2004).

${ }^{8}$ S. Takahashi, L. Zhang, S. Kobayashi, Y. Kamada, H. Kikuchi, and K. Ara, J. Appl. Phys. 98, 033909 (2005).

'J. Koutský and J. Kočík, in Radiation Damage of Structural Materials (Elsevier, New York, 1994).

${ }^{10} \mathrm{C}$. P. Steinmetz, in Theory and Calculation of Electric Circuits (McgrawHill, New York, 1917).

${ }^{11}$ S. Takahashi, H. Kikuchi, K. Ara, N. Ebine, Y. Kamada, S. Kobayashi, and M. Suzuki, J. Appl. Phys. 100, 023902 (2006). 